Communications in Physics, Vol.22, No. 1 (2012), pp. 15-31

\title{
PHASE STRUCTURE OF LINEAR SIGMA MODEL WITHOUT NEUTRALITY CONSTRAINT (I)
}

\author{
TRAN HUU PHAT \\ Vietnam Atomic Energy Commission \\ and \\ Dong Do University \\ NGUYEN VAN THU \\ Institute of Nuclear Science and Technique \\ and \\ Department of Physics, Hanoi University of Education II
}

\begin{abstract}
The pion condensation and chiral phase transition are studied within the linear sigma model with constituent quarks (LSMq). In the chiral limit the pion condensation is always the firstorder phase transition and the phase diagrams of the pion condensate are established respectively in the $(\mu, T),\left(\mu_{I}, T\right)$ and $\left(\mu_{I}, \mu\right)$-planes, here $T, \mu$ and $\mu_{I}$ are temperature, baryon chemical and isospin chemical potentials. In the physical world, where the chiral symmetry is explicitly broken we investigate systematically the phase structure of pion and chiral condensates in the $\left(T-\mu-\mu_{I}\right)$ space. The obtained results are mainly compared to the existing data derived from the lattice $Q C D$ (LQCD) and the Polyakov-loop extended Nambu-Jona-Lasinio (PNJL) model.
\end{abstract}

\section{INTRODUCTION}

It is known that the study of the phase structure of QCD turns out to be a very hot subject attracted more and more attention, both experimentally and theoretically [1]. Many aspects of in-medium effects such as the chiral restoration at high baryon density, the in-medium properties of hadrons, the pion condensation in dense matter and so on are of special interest. The high energy heavy-ion collisions are presently the powerful machinery to generate hot and dense hadronic matter, and, therefore, they create a good chance for exploring the phase structure of QCD at extreme conditions. It is commonly accepted that the chiral restoration phase transition accompanies the confinement-deconfinement phase transition at the same critical temperature. The recent experimental data of RHIC, collected for high-temperature and low-baryon density region, provide clear signals on the confinement-deconfinement phase transition. This region is also one of the research problems to be carried out at LHC. The experimental research in high-baryon density region is planned to be implemented in the future heavy-ion collision experiments at RHIC, FAIR and NICA.

The first principle calculations of lattice QCD at finite baryon density is rather problematic due to the sign puzzle. In this respect, one has to invoke different effective models to address this issue. 
We know that heavy nuclei can be considered as nuclear matter with finite isospin density due to Coulomb interactions and the isospin-dependent quantities of isotopically asymmetric nuclear matter, such as the in-medium nucleon-nucleon potential, the equations of state and, in particular, the nuclear symmetry energy plays very important role in understanding a lot of interesting astrophysical problems [2]- [4], dynamics of heavy-ion reactions at intermediate energies [5]- [8], the structure of neutron-rich nuclei, and the nuclei close to the drip-line [9]- [14]. Hence, dealing with QCD at finite isospin chemical potential is of special significance, too. The LQCD simulations $[15,16]$ at finite isospin chemical potential, finite temperature and low baryonic density were smoothly implemented and conjectured that the charged pions condense at high isospin density and low temperature, and also suggested that the transition to a Bose-Einstein condensed state coincides with the deconfinement phase transition to quark-gluon plasma at low isospin chemical potential. The pion condensation and the related phase diagrams of two-flavor QCD have been investigated by means of various QCD effective models: chiral perturbation theory [17][19], NJL-type models [20]- [23], linear sigma model [24]. We are aware that, among theories developed so far, the LQCD is the unique one based on first principle and the PNJL model is an extended version of the NJL model which respects approximately both mechanisms: chiral symmetry breaking and confinement. In this regard, perhaps, they provide a guideline for all studies of the phase structure.

Nowadays, the holographic QCD [25] emerges as a promised theory that is hopeful to explain satisfactorily all phenomena related to the non- perturbative region of QCD.

In the present paper the phase structure of hot quark matter at finite isospin chemical potential is studied systematically within the linear sigma model with two light quarks and for not too high values of baryonic chemical potential, so the di-quark condensation might be ignored. We will closely follow Ref. [26] to consider the system given by the Lagrangian

$$
\begin{aligned}
& L=\bar{q}\left[i \gamma^{\mu} \partial_{\mu}-m_{q}-g\left(\sigma+i \gamma^{5} \vec{\tau} \vec{\pi}\right)\right] q+\mu \bar{q} \gamma^{0} q+L_{L S M}, \\
& L_{L S M}=\frac{1}{2}\left[\partial_{\alpha} \sigma \partial^{\alpha} \sigma+\partial_{\alpha} \vec{\pi} \partial^{\alpha} \vec{\pi}\right]+2 i \mu_{I}\left(\pi_{1} \partial_{0} \pi_{2}-\pi_{2} \partial_{0} \pi_{1}\right)-U, \\
& U=\frac{m^{2}}{2}\left(\sigma^{2}+\vec{\pi}^{2}\right)-\frac{\mu_{I}^{2}}{2}\left(\pi_{1}^{2}+\pi_{2}^{2}\right)+\frac{\lambda^{2}}{4}\left(\sigma^{2}+\vec{\pi}^{2}\right)^{2}-\epsilon f_{\pi} m_{\pi}^{2} \sigma,
\end{aligned}
$$

where $q, \sigma$ and $\vec{\pi}$ are respectively the field operators of quarks, sigma meson and pion; $m_{\pi}$ and $f_{\pi}$ are respectively pion mass and pion decay constant in vacuum; $\mu$ and $\mu_{I}$ denote the baryon and isospin chemical potentials, $m_{q}$ is current quark mass and $\epsilon=0,1$.

The paper is structured as follows. In Section II the chiral restoration and the pion condensation of the system (1) are considered, respectively, in the chiral limit and the physical world. The conclusion and discussion are given in Section III.

\section{CHIRAL RESTORATION AND PION CONDENSATION}

At first let us establish the grand partition function

$$
Z=\int \mathcal{D} \bar{q} \mathcal{D} q \mathcal{D} \sigma \mathcal{D} \vec{\pi} \exp \int_{\beta} L
$$


here

$$
\int_{\beta}=i \int_{0}^{1 / T} d \tau \int d^{3} \vec{x}
$$

Assume that the $\sigma$ and $\vec{\pi}$ field operators develop the ground state expectation values

$$
\langle\sigma\rangle=u,\left\langle\pi_{1}^{2}+\pi_{2}^{2}\right\rangle=v^{2},\left\langle\pi_{3}\right\rangle=0 .
$$

Following the convention of choosing $\left\langle\pi_{1}\right\rangle=v,\left\langle\pi_{2}\right\rangle=0$ the isospin symmetry is spontaneously broken and a Goldstone boson appears.

In the mean field approximation applied to the meson sector $Z$ takes the form

$$
Z=\exp \left(-\frac{V U}{T}\right) \int \mathcal{D} \bar{q} \mathcal{D} q\left\{\bar{q} S^{-1}(k) q\right\}
$$

where $V$ is the system volume and

$$
\begin{aligned}
& S^{-1}(k)=\left(A_{i j}\right), \\
& A_{11}=\gamma^{\mu} k_{\mu}+\mu_{u} \gamma^{0}+g u, A_{12}=i g v \tau_{1} \gamma^{5}=A_{21}, \\
& A_{22}=\gamma^{\mu} k_{\mu}+\mu_{d} \gamma^{0}+g u
\end{aligned}
$$

with $\tau_{1}$ being the first component of the Pauli matrices.

The effective potential in one-loop approximation is derived directly from (2),

$$
\Omega(u, v)=-\frac{T \ln Z}{V}=U(u, v)+\Omega_{q \bar{q}},
$$

in which

$$
\Omega_{q \bar{q}}=\ln \operatorname{det} S^{-1}(k)=-\nu_{q} \int \frac{d^{3} \vec{k}}{(2 \pi)^{3}}\left\{E+T\left[\ln \left(1+e^{\beta E_{1}}\right)+\ln \left(1+e^{-\beta E_{2}}\right)\right]\right\} .
$$

The energy spectrum is easily obtained

$$
E=\sqrt{\vec{k}^{2}+\left(m_{q}+g u\right)^{2}+g^{2} v^{2}}, E_{1}=E+\mu, E_{2}=E-\mu, \mu_{u}+\mu_{d}=\mu=\frac{\mu_{B}}{3},
$$

$\mu_{B}$ is the baryon chemical potential.

Neglecting the divergent part in (4) and integrating out it by parts we arrive at

$$
\Omega_{\bar{q} q}=-\frac{\nu_{q}}{6 \pi^{2}} \int_{0}^{\infty} d k k^{4} \frac{n_{q}(T, \mu)+n_{\bar{q}}(T, \mu)}{E},
$$

where

$$
n_{q}(T, \mu)=\left[1+\exp \frac{1}{T}(E-\mu)\right]^{-1}, n_{\bar{q}}(T, \mu)=\left[1+\exp \frac{1}{T}(E+\mu)\right]^{-1} .
$$

The ground state of the theory is determined by requiring that

$$
\frac{\partial \Omega}{\partial u}=0, \frac{\partial \Omega}{\partial v}=0
$$


or

$$
\begin{aligned}
& {\left[m^{2}+\lambda^{2}\left(u^{2}+v^{2}\right)\right] u-\epsilon f_{\pi} m_{\pi}^{2}+\frac{\partial \Omega_{q \bar{q}}}{\partial u}=0,} \\
& {\left[-\mu_{I}^{2}+m^{2}+\lambda^{2}\left(u^{2}+v^{2}\right)\right] v+\frac{\partial \Omega_{q \bar{q}}}{\partial v}=0 .}
\end{aligned}
$$

Eqs. (7) and (8) are exactly the gap equations which can be rewritten in another forms after some analytical manipulations

$$
\begin{aligned}
\frac{\partial \Omega_{q \bar{q}}}{\partial u} & =\nu_{q} g\left(m_{q}+g u\right) \int \frac{d^{3} \vec{k}}{(2 \pi)^{3}} \frac{n_{q}(T, \mu)+n_{\bar{q}}(T, \mu)}{E}, \\
\frac{\partial \Omega_{q \bar{q}}}{\partial v} & =\nu_{q} g^{2} v \int \frac{d^{3} \vec{k}}{(2 \pi)^{3}} \frac{n_{q}(T, \mu)+n_{\bar{q}}(T, \mu)}{E} .
\end{aligned}
$$

Based on the pressure $P$ defined by

$$
P=-\left.\Omega(u, v)\right|_{\text {taken at minimum }}
$$

the isospin and flavor densities are derived straightforwardly

- Isospin density

$$
\rho_{I}=\mu_{I} v^{2} .
$$

- Flavor density

$$
\rho=\frac{\nu_{q}}{6 \pi^{2}} \int d k k^{2}\left[n_{q}(T, \mu)-n_{\bar{q}}(T, \mu)\right] .
$$

It is evident that the quark densities fulfill the equality

$$
\rho_{u}+\rho_{d}=\rho .
$$

In order to proceed to the numerical computation the values of the model parameters are usually chosen as $m_{q}=5.5 \mathrm{MeV}, f_{\pi}=93 \mathrm{MeV}, m_{\pi}=138 \mathrm{MeV}$, sigma mass $m_{\sigma}=500$ $\mathrm{MeV}$ and the coupling constant $g$ is determined to be $g=3.3$ by requiring that the mass of constituent quarks $m_{\text {constituent }}$ satisfies the equality

$$
m_{\text {constituent }}=M(T=0, \mu=0)=313 \mathrm{MeV},
$$

where

$$
M=m_{q}+g u .
$$

Now we are ready to implement the numerical study in two different cases with the aid of Mathematica [27] 


\section{II.1. In chiral limit $\epsilon=0$}

It is known [26] that in this case $\langle\sigma\rangle=u=0, m_{\pi}=0,\left\langle\pi_{1}^{2}+\pi_{2}^{2}\right\rangle=v^{2} \neq 0,\left\langle\pi_{3}\right\rangle=0$ and the chiral symmetry is explicitly broken down to $O(2)$. Following the convention of choosing the ground state to have $\left\langle\pi_{1}\right\rangle=v,\left\langle\pi_{2}\right\rangle=0$, the $O(2)$ symmetry is spontaneously broken and therefore there exists only one gap equation related to the pion condensate $v$,

$$
-\mu_{I}^{2}+m^{2}+\lambda^{2} v^{2}+\nu_{q} g \int \frac{d^{3} \vec{k}}{(2 \pi)^{3}} \frac{n_{q}(T, \mu)+n_{\bar{q}}(T, \mu)}{E}=0 .
$$

The ground state is a pion superfluid.

Starting from Eq.(13) we find respectively the following results:

1) The evolution of the pion condensate as a function of $\mu$ and $\mu_{I}$ at $T=100 \mathrm{MeV}$ is shown in Fig. 1(a), the transition to chirally symmetric phase is the first-order. At higher temperature the order of the transition remains unchanged, while in the NJL model [22] the phase transition becomes second-order for $T=150 \mathrm{MeV}$. From the phase diagrams of the pion condensate at several values of temperature plotted in Fig. 2(a) it easily seen that for $T=0,100 \mathrm{MeV}$ charged pions condense at any non-vanishing value of $\mu_{I}$, that is consistent with those calculated in the NJL model [20,22]. However, at $T=150 \mathrm{MeV}$ the pion condensation emerges only at $\mu_{I}=348 \mathrm{MeV}$.

2) In Fig. 1(b) we show the pion condensate as a function of $T$ and $\mu_{I}$ at $\mu=100$ $\mathrm{MeV}$. The transition from the pion condensed phase to the chirally symmetric phase is the first-order everywhere. The phase diagrams of the pion condensate in the $\left(T, \mu_{I}\right)$-plane at $\mu=0,150,300 \mathrm{MeV}$ are plotted in Fig. 2(b). All lines correspond to the first-order phase transition.

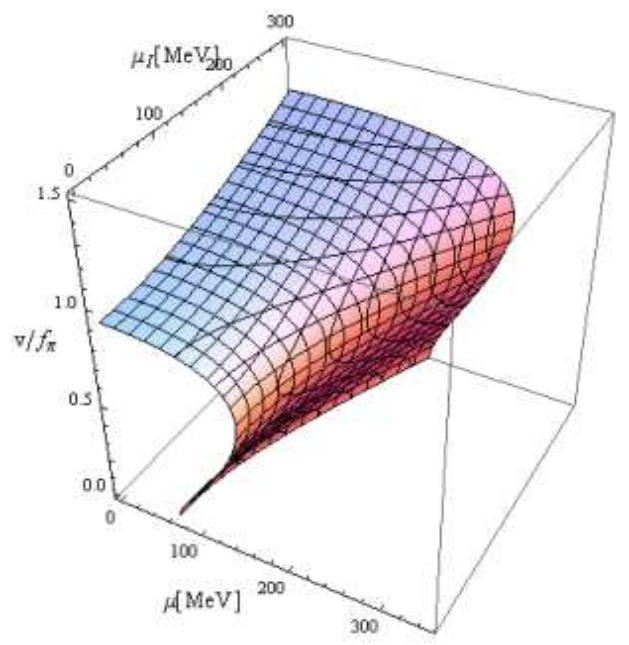

(a)

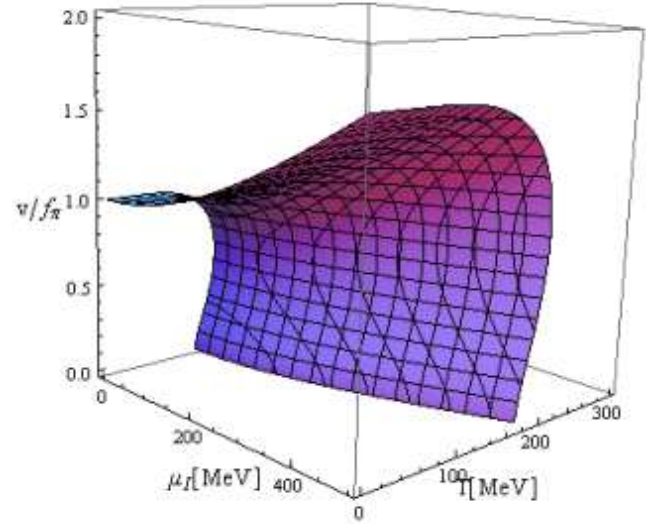

(b)

Fig. 1. The pion condensate in the chiral limit as a function: Fig. 1(a) of $\mu$ and $\mu_{I}$ at $T=100 \mathrm{MeV}$ and Fig. 1(b) of $T$ and $\mu_{I}$ at $\mu=100 \mathrm{MeV}$. 


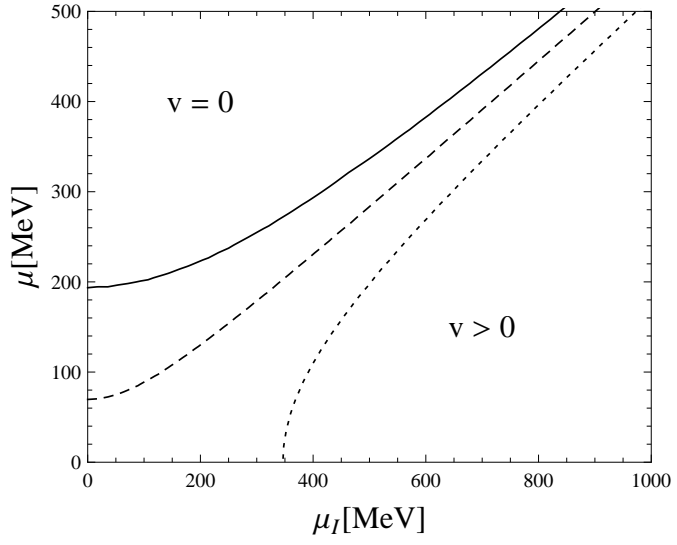

(a)

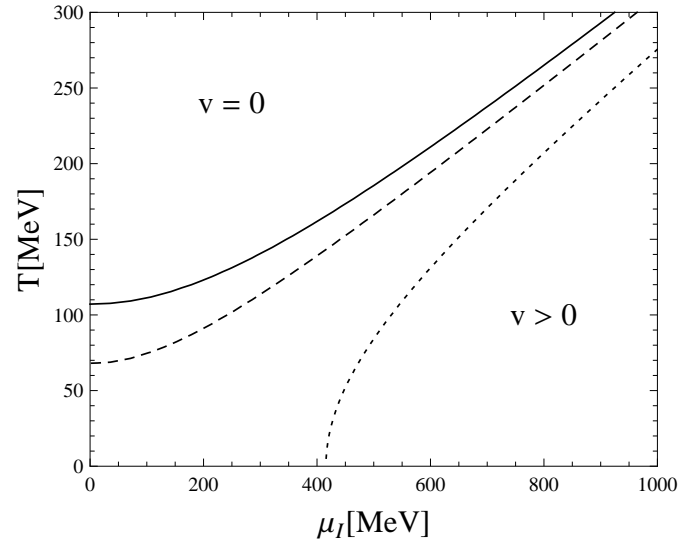

(b)

Fig. 2. The phase diagrams of pion condensate: Fig. 2(a) in the $\left(\mu, \mu_{I}\right)$-plane at $T=0$ (solid line), $100 \mathrm{MeV}$ (dashed line), $150 \mathrm{MeV}$ (dotted line). The phase transition is the first-order everywhere; Fig. $2(\mathrm{~b})$ in the $\left(T, \mu_{I}\right)$-plane at $\mu=0$ (solid line), $150 \mathrm{MeV}$ (dashed line), $300 \mathrm{MeV}$ (dotted line). The phase transition is the first-order everywhere.

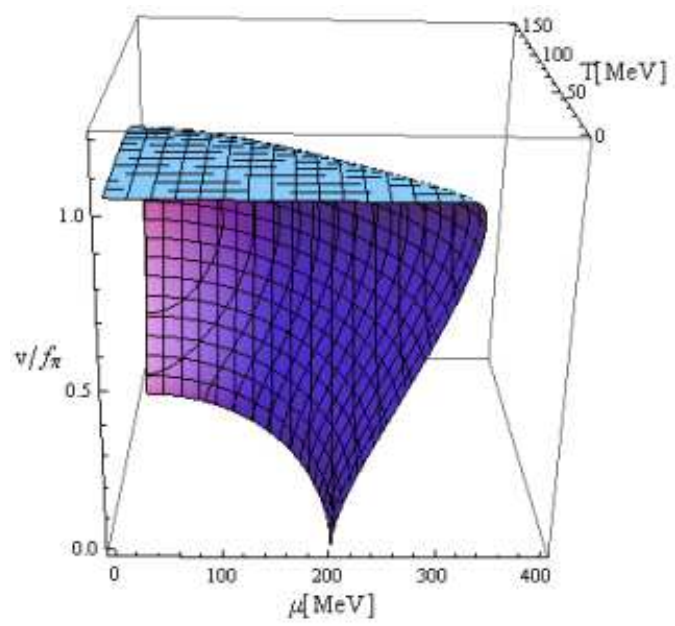

(a)

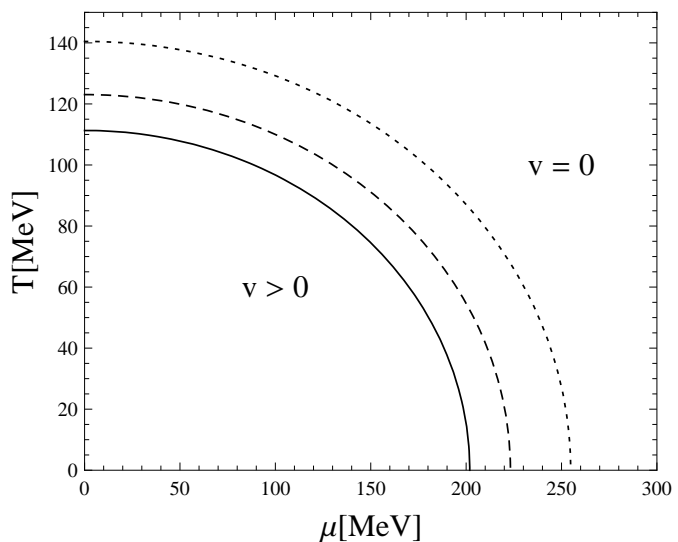

(b)

Fig. 3. The pion condensate as a function of $(T, \mu)$ at $\mu_{I}=100 \mathrm{MeV}$ in Fig. 3(a) and Fig. 3(b) is the phase diagrams of pion condensate in the $(T, \mu)$-plane at $\mu_{I}=100 \mathrm{MeV}$ (solid line), $200 \mathrm{MeV}$ (dashed line), 300MeV (dotted line). The phase transition is the first-order everywhere.

3) We show respectively in Figs. 3(a) and 3(b) the pion condensate as a function of $T$ and $\mu$ at $\mu_{I}=100 \mathrm{MeV}$ and its corresponding phase diagram in the $(T, \mu)$-plane at $\mu_{I}=100,200,300 \mathrm{MeV}$. All lines designate the first-order phase transition, whereas 
the calculations based on the NJL model [22] indicated that a first-order phase transition, starting from $T=0$, ends at a critical point given by $T=110 \mathrm{MeV}, \mu=220 \mathrm{MeV}$, while the transition is the second-order at higher $T$ and smaller $\mu$.

\section{II.2. Physical world $\epsilon=1$}

The values of $m^{2}$ and $\lambda^{2}$ are then determined by

$$
m^{2}=\frac{3 m_{\pi}^{2}-m_{\sigma}^{2}}{2}<0, \lambda^{2}=\frac{m_{\sigma}^{2}-m_{\pi}^{2}}{2 f_{\pi}^{2}}>0 .
$$

This is the case when the chiral symmetry is explicitly broken and we know [26] that there are two different ground states corresponding to $\mu_{I}<m_{\pi}$ and $\mu_{I}>m_{\pi}$, respectively:

For $\mu_{I}<m_{\pi}$,

$$
\langle\sigma\rangle=u \neq 0,\left\langle\pi_{i}\right\rangle=0, i=1,2,3 .
$$

Accordingly, we have only one gap equation

$$
\left[m^{2}+\lambda^{2} u^{2}\right] u-f_{\pi} m_{\pi}^{2}+\nu_{q} g\left(m_{q}+g u\right) I=0,
$$

with

$$
I=\int \frac{d^{3} \vec{k}}{(2 \pi)^{3}} \frac{n_{q}(T, \mu)+n_{\bar{q}}(T, \mu)}{E} .
$$

For $\mu_{I}>m_{\pi}$

$$
\langle\sigma\rangle=u \neq 0,\left\langle\pi_{1}^{2}+\pi_{2}^{2}\right\rangle=v^{2} \neq 0,\left\langle\pi_{3}\right\rangle=0 .
$$

Choosing $\left\langle\pi_{1}\right\rangle=v,\left\langle\pi_{2}\right\rangle=0$ the isospin symmetry is spontaneously broken and a Goldstone boson appears. The corresponding gap equations read

$$
\begin{aligned}
& {\left[m^{2}+\lambda^{2}\left(u^{2}+v^{2}\right)\right] u-f_{\pi} m_{\pi}^{2}+\frac{\partial \Omega_{q \bar{q}}}{\partial u}=0,} \\
& {\left[-\mu_{I}^{2}+m^{2}+\lambda^{2}\left(u^{2}+v^{2}\right)\right] v+\frac{\partial \Omega_{q \bar{q}}}{\partial v}=0,}
\end{aligned}
$$

in which

$$
\begin{aligned}
\frac{\partial \Omega_{q \bar{q}}}{\partial u} & =\nu_{q} g\left(m_{q}+g u\right) \int \frac{d^{3} \vec{k}}{(2 \pi)^{3}} \frac{n_{q}(T, \mu)+n_{\bar{q}}(T, \mu)}{E}, \\
\frac{\partial \Omega_{q \bar{q}}}{\partial v} & =\nu_{q} g^{2} v \int \frac{d^{3} \vec{k}}{(2 \pi)^{3}} \frac{n_{q}(T, \mu)+n_{\bar{q}}(T, \mu)}{E} .
\end{aligned}
$$

Now the numerical study is first devoted to the case $\mu_{I} \geq m_{\pi}$, then both chiral and pion condensates are non-vanishing. Solving simultaneously the gap equations (16) we obtain the following results:

1) The pion condensation and the chiral restoration at $T=\mu=0$ are represented in Fig. 4(a), which shows the competition between two condensates: the chiral condensate is constant in the interval $0 \leq \mu_{I} \leq m_{\pi}$ and at $\mu_{I}=m_{\pi}$ the chiral symmetry gets restored for large $\mu_{I}$. This behavior is in agreement with the LQCD $[15,16]$. Meanwhile, the charged pions condense for $\mu_{I} \geq m_{\pi}$ and the pion condensate increases infinitely for growing $\mu_{I}$. The behavior of the pion condensate in our calculation is basically in agreement with the 
one suggested in [17] at large values of $\mu_{I}$. Of course, the unbounded growth of pion condensate as $\mu_{I}$ increases is physically un-acceptable.

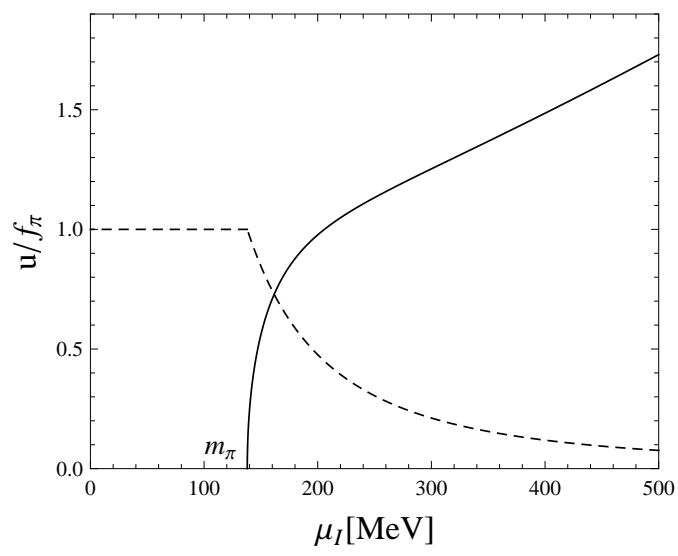

(a)

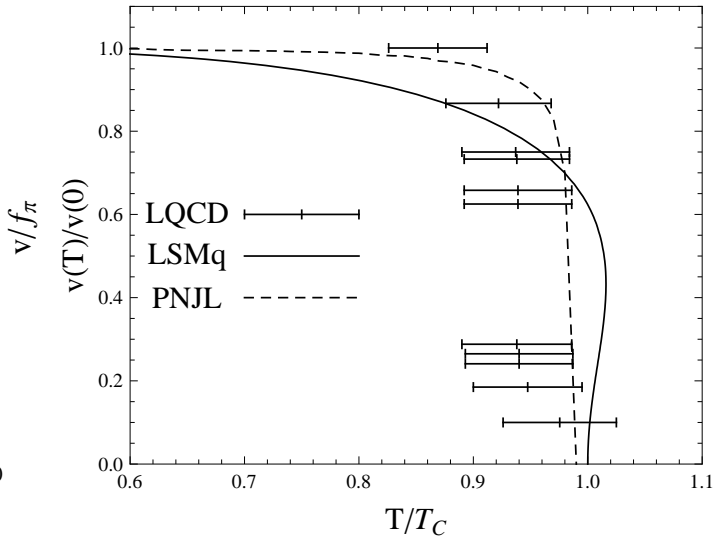

(b)

Fig. 4. The evolutions of chiral (dashed line) and pion (solid line) condensates as functions of $\mu_{I}$ at $T=\mu=0$ in Fig. 4(a) and in Fig. 4(b) the pion condensate as a function of $T$ at $\mu=0$ and $\mu_{I}=192 \mathrm{MeV}$. The critical temperature $T_{c}=128.73$ $\mathrm{MeV}$. The phase transition is the first-order.

2) Various facets of the pion condensation in different regions of three parameters $\mu_{I}, \mu$ and $T$ are displayed as follows.

a) In Fig. 5(a) we show the pion condensate as a function of $T$ and $\mu_{I}$ at $\mu=0$. In order to compare with other works let us consider the evolutions of the pion condensate against $T$ at $\mu=0$ and several $\mu_{I}$ steps and the corresponding phase diagram in the $\left(\mu_{I}, T\right)$-plane. They are represented respectively in Figs. 4(b) and 6(a). Fig. 4(b) indicates that the results found here are close to the data derived from the LQCD [15] and PNJL model [23]. Fig. 6(a) shows that, in contrast to numerical computations in the NJL model and the chiral perturbation theory $[17,18,22]$, the system undergoes both first and secondorder phase transitions: the line of first-order transition (solid line) ends at a tri-critical point $\mathrm{C}\left(T=128.3 \mathrm{MeV}, \mu_{I}=181 \mathrm{MeV}\right)$ and the line of second-order phase transition (dashed line) extends down to $T=0, \mu_{I}=m_{\pi}$. This scenario of the phase transitions is in agreement with the LQCD simulations [15] and PNJL model [23]. In Figs. 5(b) and 6(b) are plotted respectively the pion condensate as a function of $T$ and $\mu_{I}$ at $\mu=50 \mathrm{MeV}$ and the corresponding phase diagram in the $\left(T, \mu_{I}\right)$-plane. It results that the scenario of the phase transition remains the same at non-vanishing $\mu$. This statement is confirmed by investigating the behavior of the effective potential versus $v$ at $\mu=50 \mathrm{MeV}$, Fig. 7, where the solid line characterizing a first-order phase transition possesses two minima, the one at origin corresponds to the restored phase and the other corresponds to the broken phase. As $T$ and $\mu_{I}$ decrease the barrier between two minima smears out and disappears 


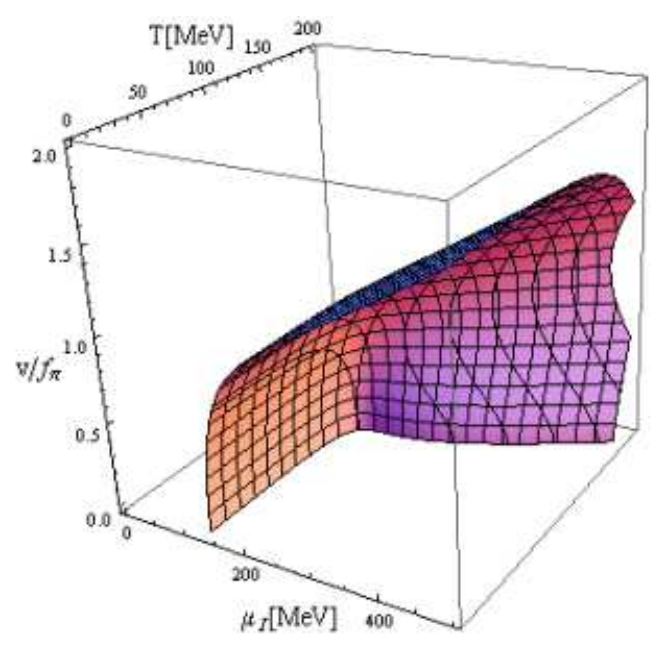

(a)

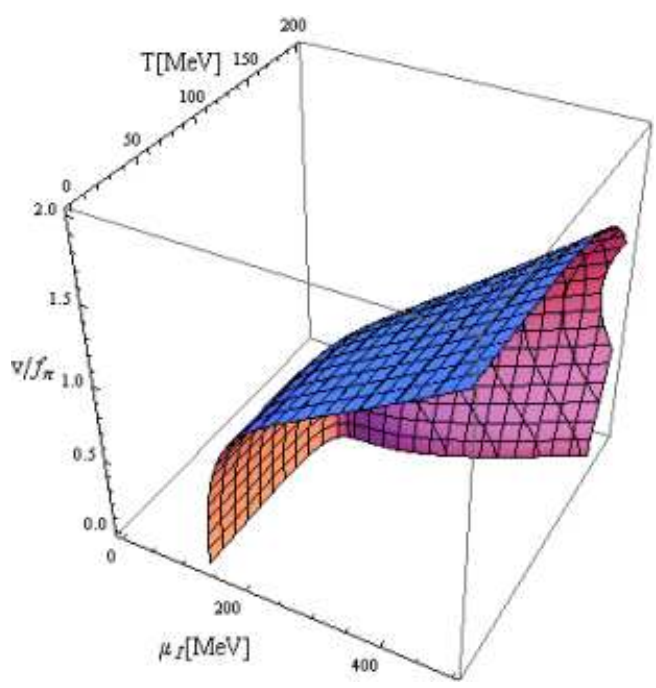

(b)

Fig. 5. The pion condensate as a function: Fig. 5(a) of $T$ and $\mu_{I}$ at $\mu=0$ and Fig. 5(b) of $T$ and $\mu_{I}$ at $\mu=50 \mathrm{MeV}$.

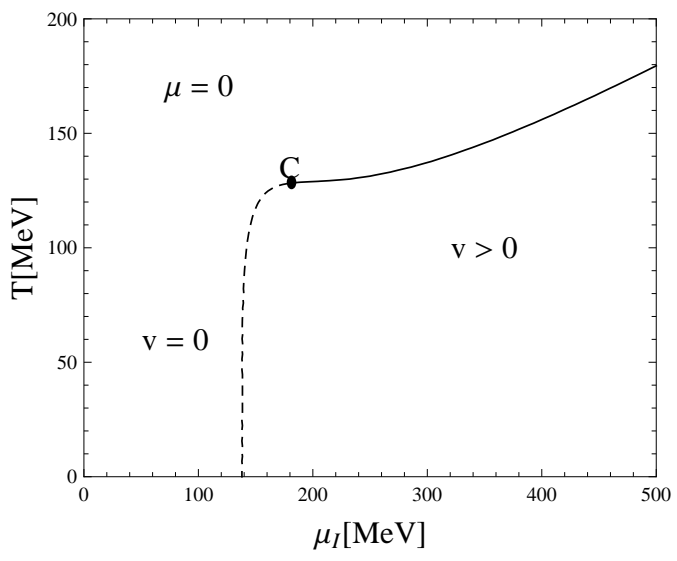

(a)

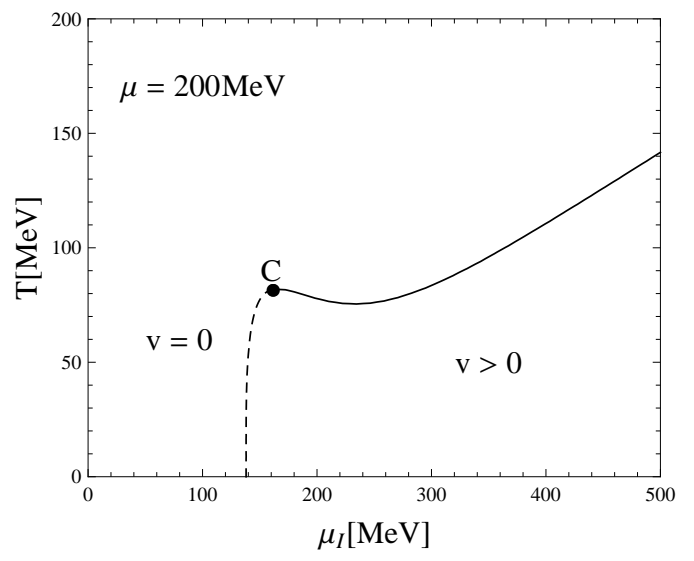

(b)

Fig. 6. The phase diagram of pion condensate in the $\left(T, \mu_{I}\right)$-plane: Fig. 6(a) at $\mu=0$. There exhibit a second-order phase transition (dashed line) and a first-order phase transition (solid line) separated by a tri-critical point C (181 $\mathrm{MeV}, 128.3 \mathrm{MeV}$ ) and Fig. 6(b) at $\mu=200 \mathrm{MeV}$. The solid line (dashed line) denotes first-order (second-order) phase transitions. C ( $162.1 \mathrm{MeV}, 81.8 \mathrm{MeV})$ is the tri-critical point.

at $\left(T, \mu_{I}, T\right)=(178.4,127.8) \mathrm{MeV}$, signaling the onset of second-order phase transition featured by a line with flattest minimum. 


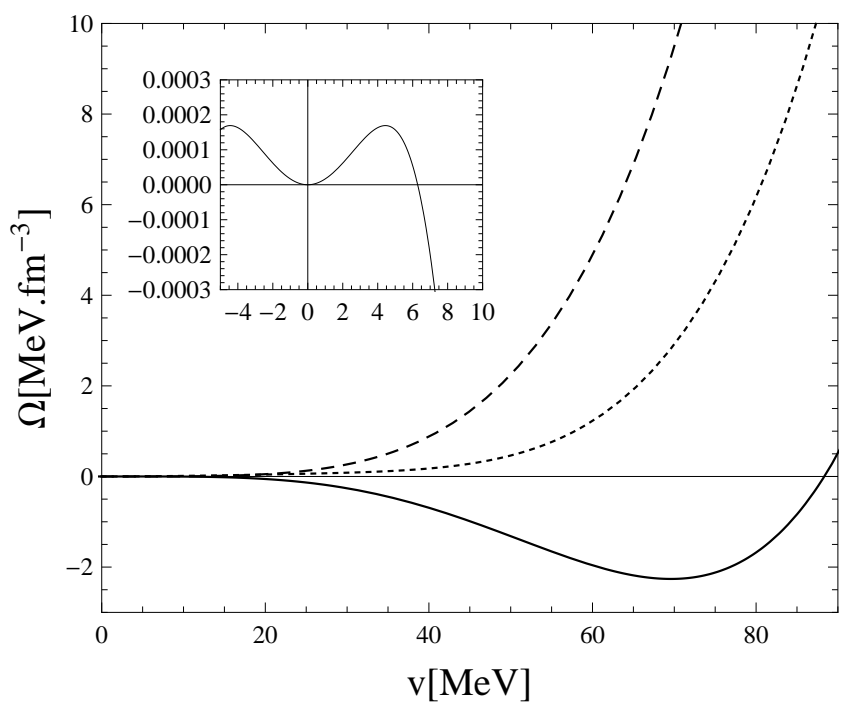

Fig. 7. The behavior of effective potential versus $v$ at $\mu=50 \mathrm{MeV}$. The solid line characterizing the first-order phase transition possesses two minima, the one at origin corresponds to restored phase and the other corresponds to broken phase. The barrier between two minima smears out as $T$ decreases and disappears at $T=114.8 \mathrm{MeV}$, a second-order phase transition appears which corresponds to the a line with flattest minimum. The insert shows the second minimum at origin.

b) In Fig. 8(a) is shown the pion condensate as a function of $\mu$ and $\mu_{I}$ at $T=0$, where from we find the phase diagram in the $\left(\mu, \mu_{I}\right)$-plane given in Fig. 9(a) which manifests the scenario: the line of first-order phase transition ends at a tri-critical point $\mathrm{C}$ and the line of second-order transition extends down to $\mu=0, \mu_{I}=m_{\pi}$. Qualitatively, this phase diagram is similar to the figures 3,6 and 7 of Refs. [22,28] and [23], accordingly. At higher values of temperature, $T=50,100,150 \mathrm{MeV}$, the corresponding phase diagrams in the $\left(\mu, \mu_{I}\right)$-plane are depicted respectively in Figs. 10(a), 10(b) and 10(c). We see that starting from $T=150 \mathrm{MeV}$ the phase diagram gets a crucial change: the pion condensation turns out to be first-order everywhere.

c) In Figs. 8(b) and 9(b) we show the pion condensate as a function of $T$ and $\mu$ at $\mu_{I}=150 \mathrm{MeV}\left(>m_{\pi}\right)$ and its phase diagram in the $(T, \mu)$-plane at $\mu_{I}=150 \mathrm{MeV}$. The solid line (dashed line) denotes first-order (second-order) phase transition. $\mathrm{C}$ ( $\mathrm{T}=$ $34 \mathrm{MeV}, \mu=862.55 \mathrm{MeV}$ ) is the tri-critical point. Our phase diagram is basically similar to Fig. 13 of Ref. [23].

3) Next let us go to the study of chiral restoration whose phenomenological issues and possible experimental signatures were discussed by Harris and Muller [29].

a) Firstly we begin with the case $\mu_{I}<m_{\pi}$, where the ground state corresponds to $v=0$ and $u$ determined by the gap equation (15). In Figs. 11(a) and 11(b) we show respectively the dependences of $u / f_{\pi}$ as a function of $\mu$ for various values of $T$ and function of $T$ for several values of $\mu$. It is obvious that the chiral symmetry gets restored at high baryonic density for corresponding values $T$ and/or at high temperature for corresponding 


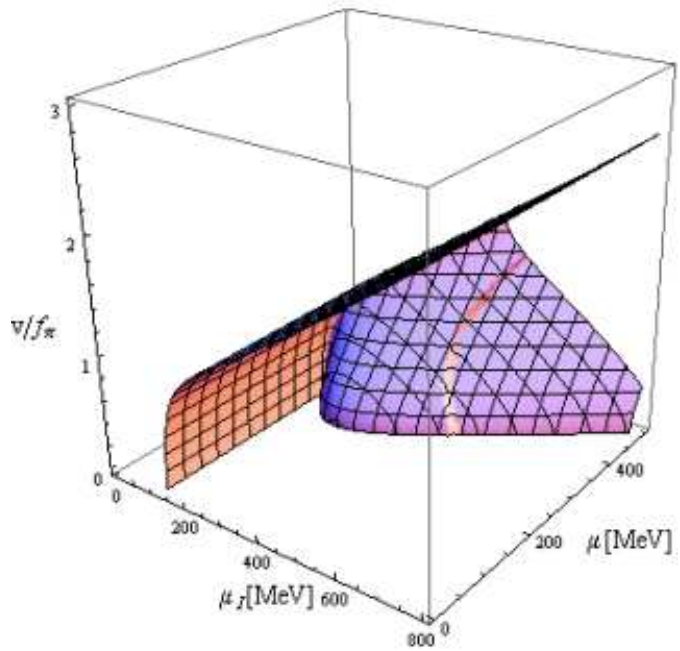

(a)

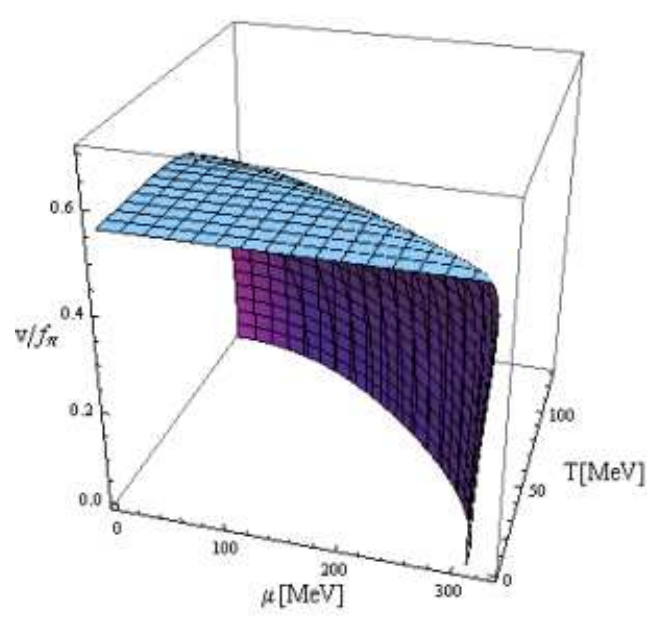

(b)

Fig. 8. The pion condensate as a function: Fig. 8(a) of $\mu$ and $\mu_{I}$ at $T=0$; Fig. $8(\mathrm{~b})$ of $T$ and $\mu$ at $\mu_{I}=150 \mathrm{MeV}$.

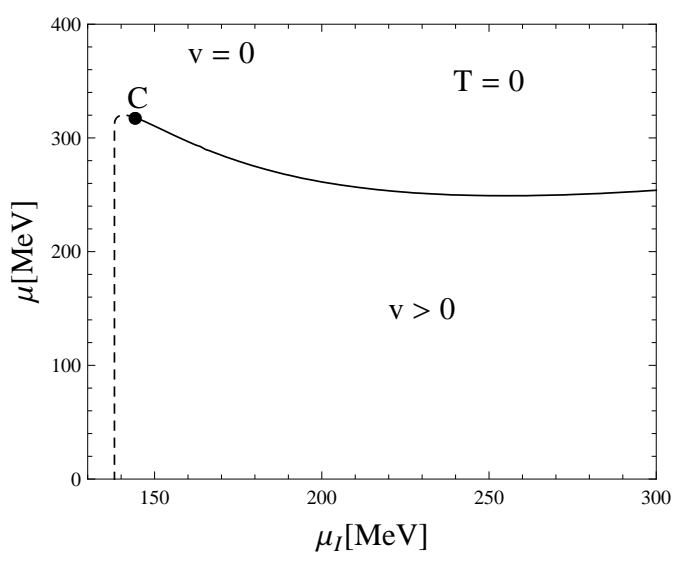

(a)

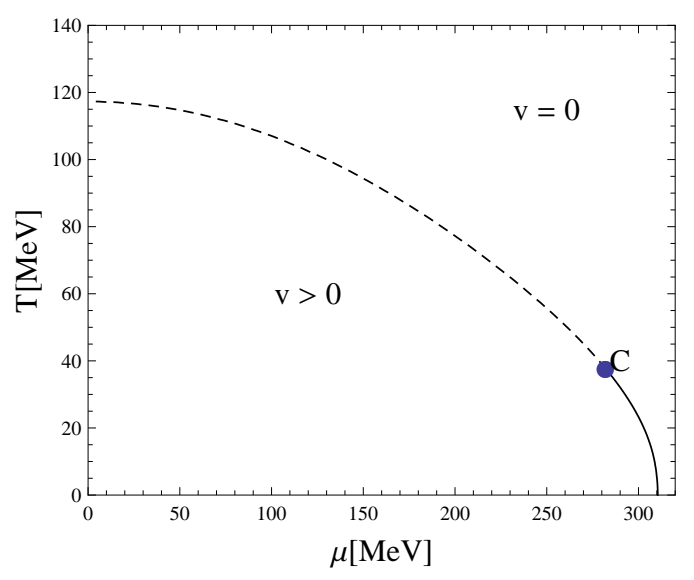

(b)

Fig. 9. The phase diagram of pion condensate: Fig. 9(a) in the $\left(\mu, \mu_{I}\right)$-plane at $T=0$ with $\mathrm{C}(144.3 \mathrm{MeV}, 317.7 \mathrm{MeV})$; Fig. $9(\mathrm{~b})$ in the $(\mu, T)$-plane at $\mu_{I}=150$ $\mathrm{MeV}$ with $\mathrm{C}(282 \mathrm{MeV}, 37.7 \mathrm{MeV})$. The solid and dashed lines correspond to the first and second-order phase transition.

values of $\mu$. The smooth behaviors of the chiral condensate as function of temperature or density lead several authors to believe that the phase transition is second-order [30]. As mentioned in Ref. [31], in reality solving only the gap equation is not sufficient to decide whether the phase transition is first or second-order. In this connection, several methods 


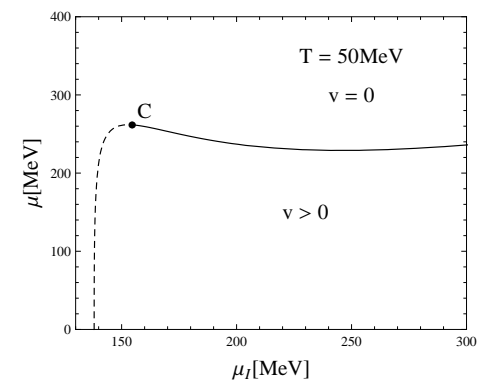

(a)

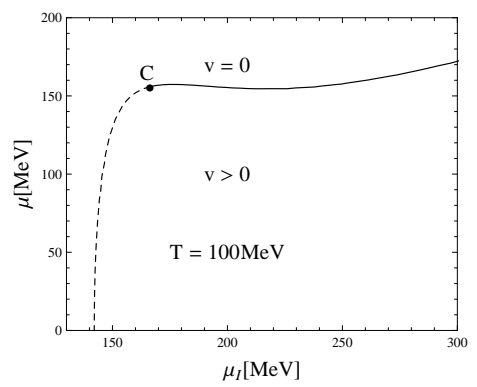

(b)

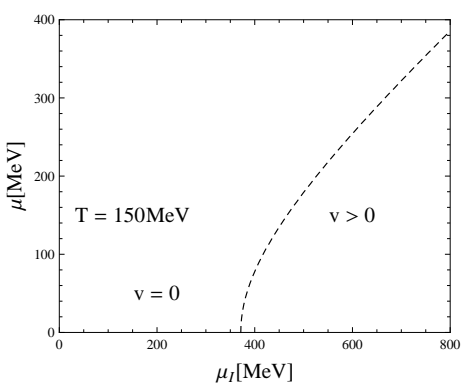

(c)

Fig. 10. The phase diagram of pion condensate in the $\left(\mu, \mu_{I}\right)$ plane at $T=50$ $\mathrm{MeV}$ (Fig. 10(a)), $100 \mathrm{MeV}$ (Fig. 10(b)). The solid (dashed) line means first (second)-order phase transition. $\mathrm{C}$ is the tri-critical point. At $T=150 \mathrm{MeV}$ (Fig. 10(c)) the second-order phase transition never occurs, only first-order phase transition is present.

have been made use to determine the order of the chiral restoration phase transition in NJL [32] as well as linear sigma models. From Fig. 11(b) it is easily recognized that there exist two regions :a) for $\mu<100 \mathrm{MeV}$ the order parameter $u$ is a single-valued function of $T$ as tending toward zero. b) for $\mu>100 \mathrm{MeV}$ the order parameter $u$ becomes a multi-valued function of $T$ as going down. We adopt here the approach based on the consideration of the evolution of effective potential $\Omega$ versus $M$. In Fig. 12(a) we show the phase diagram of the chiral condensate in the $(T, \mu)$-plane. The solid line corresponds to the state where two phases coexist in the first-order phase transition. Along this line the effective potential possesses two minima of equal depth separated by a barrier as presented in Fig. 13(a). As the temperature increases the barrier smears out gradually and eventually disappears at $T=100 \mathrm{MeV}$, signaling the onset of a second-order phase transition characterized by a line with a flattest minimum. Thus, the solid line in Fig. 12(a) ends at the second-order critical endpoint (CEP). The location of the CEP is still an open problem and has been intensively discussed $[34,35]$. There is a similarity to Fig.8 of Ref. [23].

b) Now the case $\mu_{I}>m_{\pi}$ is dealt with. The ground state is determined simultaneously by two gap equations (16). In Fig. 15 we show the evolution of chiral condensate as a function of $T$ at $\mu_{I}=150 \mathrm{MeV}$ (Fig. 14(a)), $300 \mathrm{MeV}$ (Fig. 14(b)) and several values of $\mu$. The chiral restoration is a smooth transition as defined in $[32,33]$. The evolution of chiral condensate as a function of $\mu$ at $\mu_{I}=150 \mathrm{MeV}$ and several values of $T$ is indicated in Fig. 15(a). As is seen, $u$ is a multi-valued function of $\mu$ for $0<T<100 \mathrm{MeV}$ and turns out to be single-valued for $T>100 \mathrm{MeV}$.

Applying the previous method we arrive at the phase diagram in the $(T, \mu)$-plane at $\mu_{I}=150 \mathrm{MeV}$, Fig. 12(b). Here the solid line denotes the first-order phase transition and CEP is the second-order critical endpoint. It is obvious that there is a qualitative agreement with Fig. 13 of the PNJL calculations [23]. This result is found out by computing the $M$ dependence of $\Omega(M)$ at $\mu_{I}=150 \mathrm{MeV}$ as depicted in Fig. 13(b), where the first-order phase transition is represented by a line involving two equal-depth minima with a barrier 


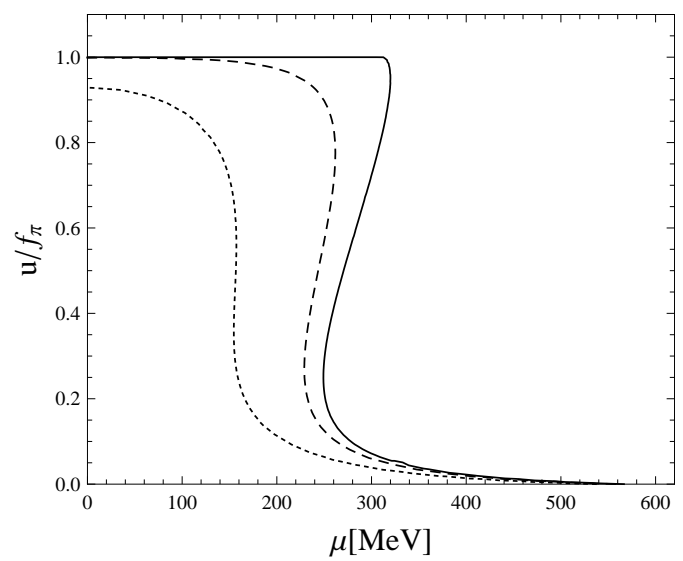

(a)

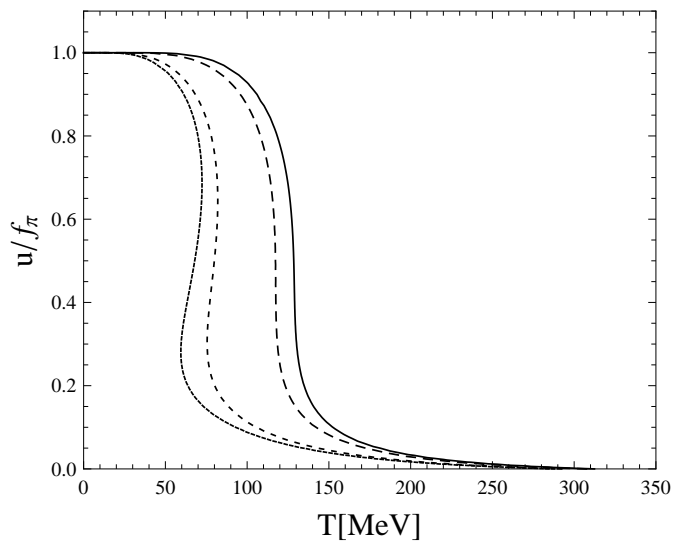

(b)

Fig. 11. The evolution of $u / f_{\pi}$ in the region $\mu_{I}<m_{\pi}$ as a function: Fig. 11(a) of $\mu$, from the top to bottom the graphs correspond to $T=0,50,100 \mathrm{MeV}$; Fig. 11(b) of $T$, from the top to bottom the graphs correspond to $\mu=0,100,200,220 \mathrm{MeV}$.

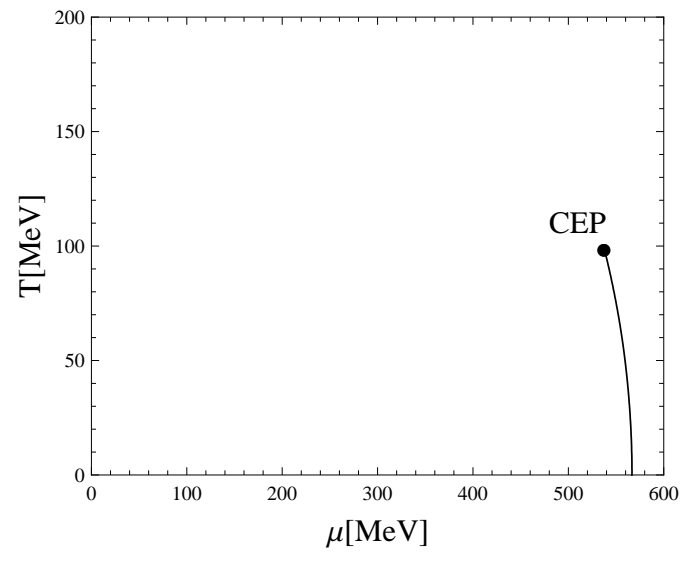

(a)

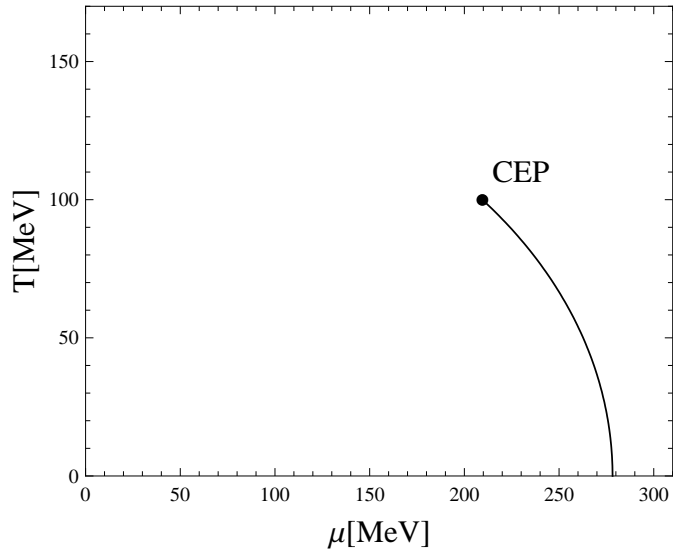

(b)

Fig. 12. The phase diagram of the chiral condensate in the $(T, \mu)$-plane: Fig. 12(a) or $\mu_{I}<m_{\pi}$; Fig. 12(b) at $\mu_{I}=150 \mathrm{MeV}$. CEP is the critical endpoint of the first-order phase transition.

in between. As $T$ increases to $T=100 \mathrm{MeV}$ the barrier disappears, signaling the onset of a second-order phase transition featured by a line with flattest minimum. However, at larger value of $\mu_{I}$, say, $\mu_{I}=300 \mathrm{MeV}$, the $\mu$ dependence of chiral condensate given in Fig. 15(b) tells that no first-order phase transition occurs and the chiral restoration is smooth transition everywhere.

Finally, we show in Figs. 16(a) and 16(b) the $\mu_{I}$ dependence of chiral condensate at $\mu=0$ (Fig. 16(a)), $150 \mathrm{MeV}$ (Fig. 16(b)) for several temperature steps. It is clear 


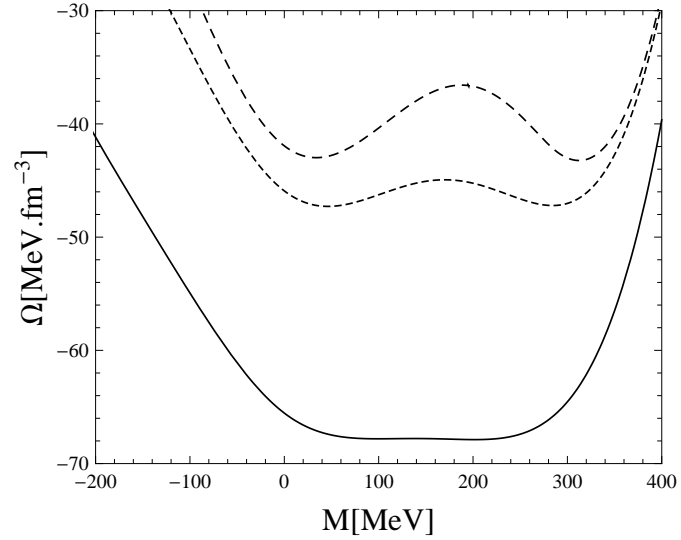

(a)

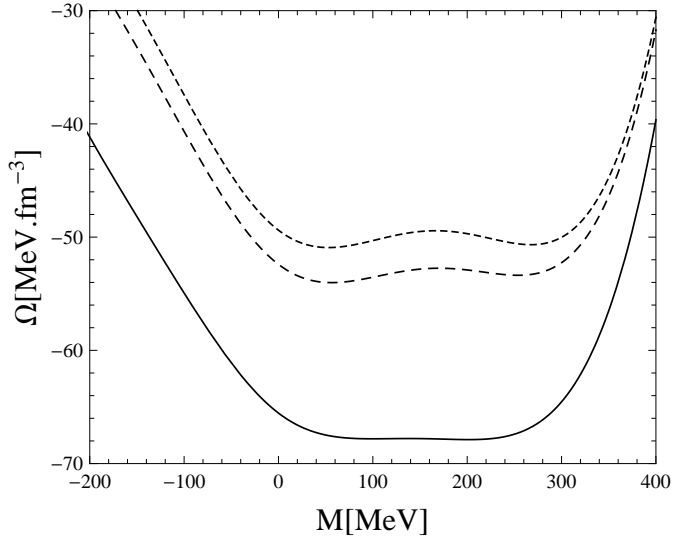

(b)

Fig. 13. The evolution of the effective potential versus the constituent mass of quark $M$ : Fig. 13(a) for $\mu_{I}<m_{\pi}$. From the top to bottom the graphs correspond to $T=0,50,100 \mathrm{MeV}$ and Fig. $13(\mathrm{~b})$ at $\mu_{I}=150 \mathrm{MeV}$. From the top the lines correspond to $T=50,70,100 \mathrm{MeV}$.

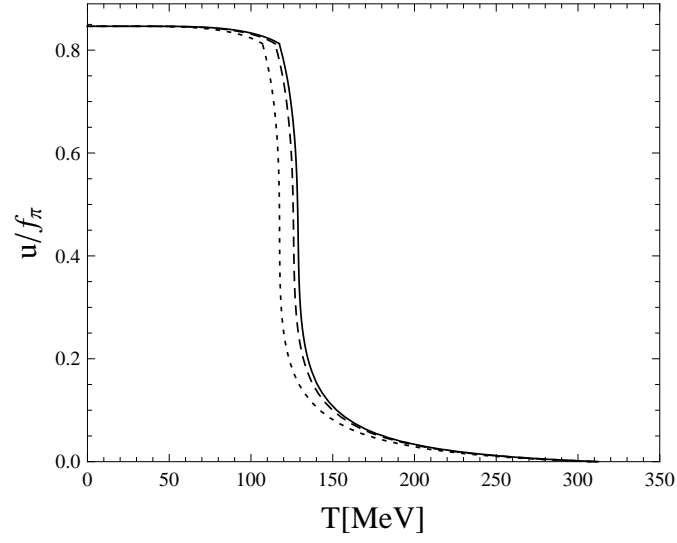

(a)

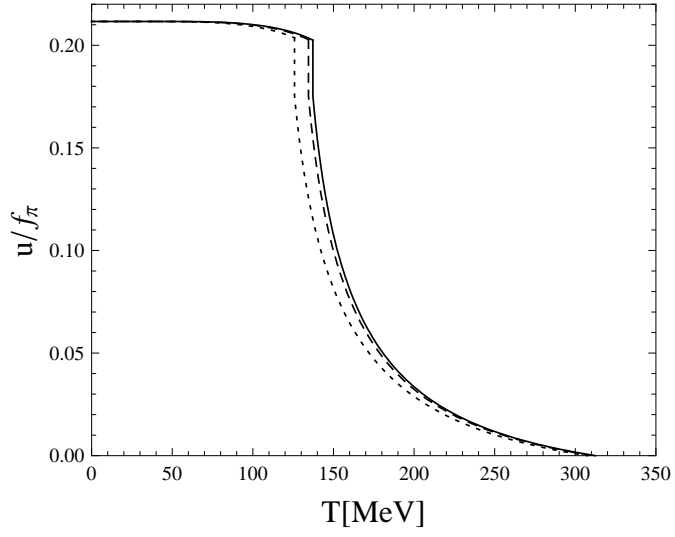

(b)

Fig. 14. The evolution of the chiral condensate as a function of $T$ at $\mu_{I}=150$ $\mathrm{MeV}$ (Fig. 14(a)), $300 \mathrm{MeV}$ (Fig. 14(b)) and $\mu=0$ (solid line), $50 \mathrm{MeV}$ (dashed line), $100 \mathrm{MeV}$ (dotted line).

that $u$ remains constant for $\mu_{I}<m_{\pi}$ and drops down robustly for higher $\mu_{I}$, the chiral symmetry gets partially restored at high isospin densities. We also show in these figures the evolutions of pion condensate versus $\mu_{I}$ at $\mu=0$ (Fig. 16(a)), $150 \mathrm{MeV}$ (Fig. 16(b)) for comparison. There manifests a competition between two condensates as $\mu_{I}$ increases. 


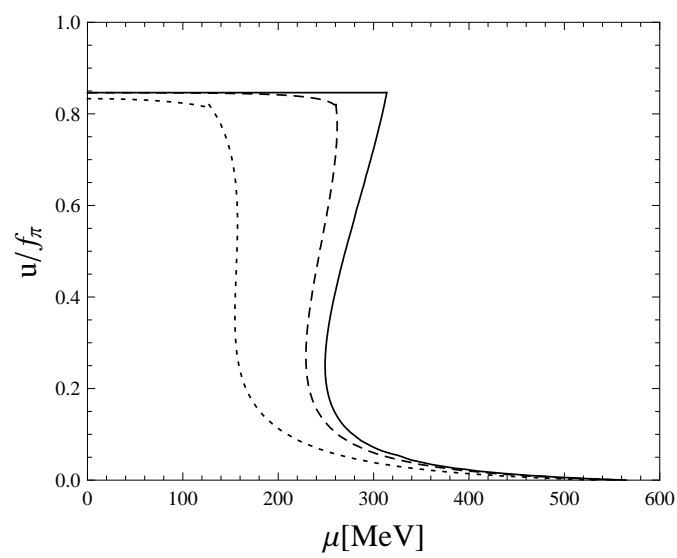

(a)

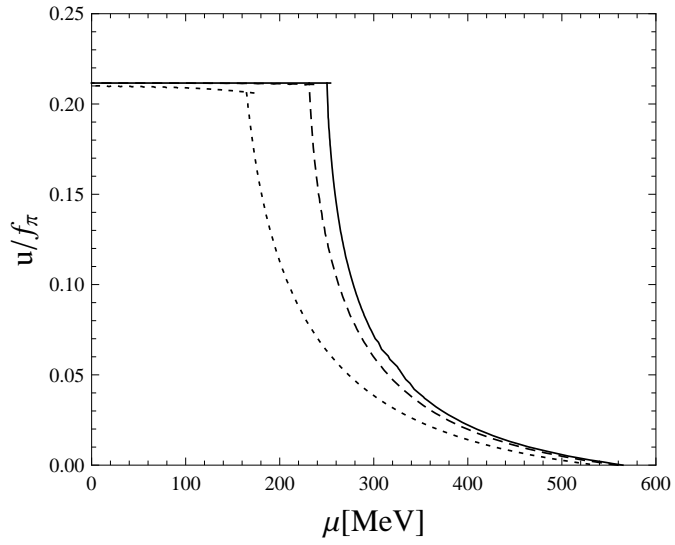

(b)

Fig. 15. The evolution of chiral condensate as a function of $\mu$ at $T=0$ (solid line), $50 \mathrm{MeV}$ (dashed line), $100 \mathrm{MeV}$ (dotted line). In Fig. 15(a) at $\mu_{I}=150$ $\mathrm{MeV}$ and Fig. $15(\mathrm{~b})$ at $\mu_{I}=300 \mathrm{MeV}$.

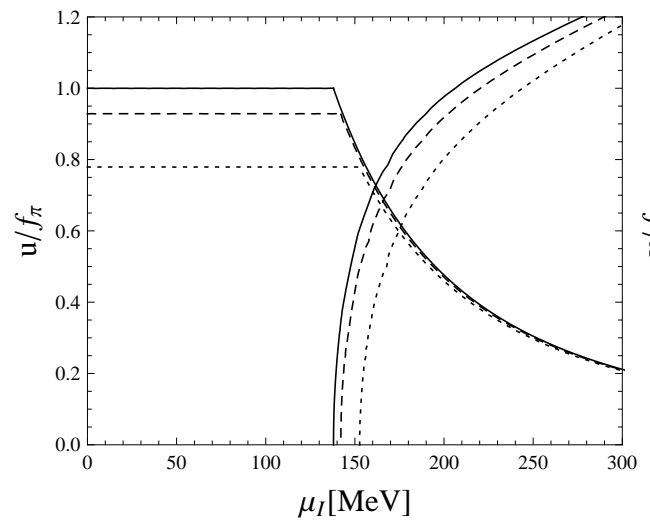

(a)

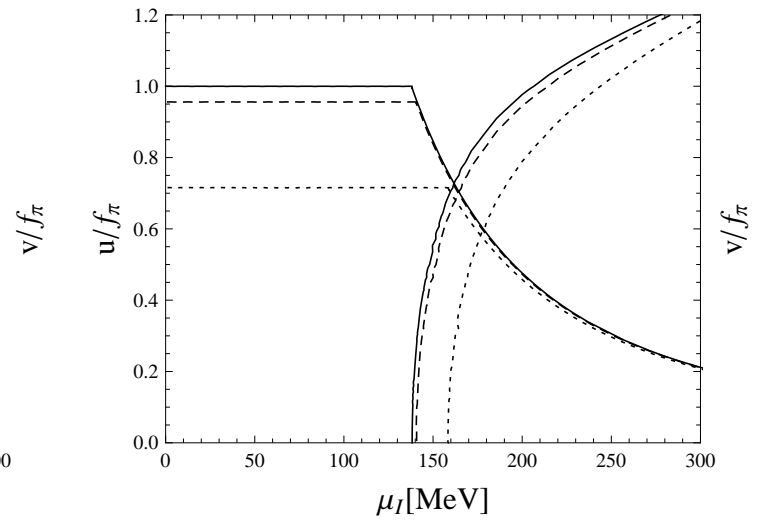

(b)

Fig. 16. The $\mu_{I}$ dependence of the chiral condensate and the pion condensate: (a) $\mu=0$ and $T=0$ (solid line), $100 \mathrm{MeV}$ (dashed line), $120 \mathrm{MeV}$ (dotted line), (b) $\mu=150 \mathrm{MeV}$ and $T=0$ (solid line), $70 \mathrm{MeV}$ (dashed line), $100 \mathrm{MeV}$ (dotted line).

\section{CONCLUSION AND DISCUSSION}

In this paper the phase structure of the linear sigma model with constituent quarks was investigated systematically and the main results are in order.

1. In the chiral limit the chiral condensate vanishes and the pion condensation is always the first-order phase transition. 
2. In the physical world the ground state is determined by the existence of both chiral and pion condensates for $\mu_{I}>m_{\pi}$ and the corresponding phase transitions were found as follows.

a) The phase diagram of the pion condensate in the $\left(T, \mu_{I}\right)$-plane at fixed $\mu$ shows that the line of first-order phase transition ends at a tri-critical point and the line of second-order phase transition extends down to $T=0$. There is a qualitative agreement with the existing data of LQCD [15] and PNJL model [23].

b) The LQCD [15], PNJL model [23] and our calculations yield qualitatively the same phase diagram of the pion condensate in the $\left(\mu, \mu_{I}\right)$ plane at zero temperature $T$ : the line of first-order phase transition ends at a tri-critical point and the line of secondorder phase transition extends down to $\mu=0$. Our calculations still conjectured that this picture remains unchanged for $T<150 \mathrm{MeV}$, but at higher $T$ the transition becomes the first-order everywhere.

c) The phase diagram of pion condensate in the $(T, \mu)$-plane at $\mu_{I}=150 \mathrm{MeV}$, Fig. 9(b), shows a similarity with Fig. 13 of Ref. [23].

d) The phase diagram of chiral condensate in the $(T, \mu)$-plane for $\mu_{I}<m_{\pi}$ expresses the fact that chiral phase transition is a first-order and ends at a second-order critical endpoint, CEP. This statement was also suggested by the PNJL model caculations [23].

In the case $\mu_{I}>m_{\pi}$ the chiral phase transition is a first-order in the $(T, \mu)$ plane and ends up at a CEP for $\mu_{I}=150 \mathrm{MeV}$. The PNJL model also provided an analogous finding, Fig. 8 of Ref. [23]. Meanwhile, for $\mu_{I}$ around $300 \mathrm{MeV}$ no first-order chiral phase transition emerges and the chiral restoration is a smooth transition everywhere.

e) The evolutions of both chiral and pion condensates as a functions of $\mu_{I}$ at vanishing and non-vanishing values of $\mu$ and $T$ manifest clearly the competition between two condensates as $\mu_{I}$ grow up.

What we presented above indicates that the linear sigma model is not expected to generate results which are in qualitative as well as quantitative agreement with those calculated by QCD since it preserves only the chiral-symmetry breaking mechanism of QCD. In addition, it is known that beside the phase transition related to chiral and pion condensates the QCD phase structure also involves the condensations of di-quarks and heavy mesons like $\omega, \rho$ and so on. In this respect, the linear sigma model would be extended to include di-quarks and heavy mesons, on the one hand, and to describe both mechanisms of QCD, chiral symmetry breaking and confinement, on the other hand. This is the Polyakov - loop extended linear sigma model. Perhaps, it is the better candidate for exploring successfully many physical properties of strongly interacting matter under extreme conditions.

\section{ACKNOWLEDGMENT}

N. V. Thu expresses his sincere thanks to the Vietnam Atomic Energy Commission and the Institute of Nuclear Science and Technique for the hospitality extended to him during his $\mathrm{PhD}$ study.

This paper is financially supported by the Vietnam National Foundation for Science and Technology Development ( NAFOSTED). 


\section{REFERENCES}

[1] M. A. Stephanov, Prog. Theor. Phys. Suppl. 153, 139 (2004); J. Wambach, Proceedings of International Workshop on Hot and Cold Baryonic Matter., Budapest, Aug. 15-20, 2010, Hungaria; D. H. Rischke, Proc. Part. Nucl. Phys. 52, 197 (2004).

[2] A. W. Steiner, M. Prakash, J. M. Lattimer, and P. J. Ellis, Phys. Rept. 411 (2005) 325.

[3] C. H. Hee, Phys. Rept. 275, 255 (1996).

[4] I. Bombaci, in Isospin Physics in Heavy-Ion Collisions, edited by B. A. Li and Udo Schroeder, Nova Science Publishers, Inc., New York, 2001, pp.35-81.

[5] V. Baran, M. Colonna, V. Greco, and M. Di Toro, Phys. Rept. 410 (2005) 335.

[6] B. A. Li, L. W. Chen, and C. M. Ko, Phys. Rept. 464 (2008) 113.

[7] P. Danielewicz and Jenny Lee, Int. J. Mod. Phys. E18 (2009) 892.

[8] B. A. Li, C. M. Ko, and W. Bauer, Int. J. Mod. Phys. E7 (1998) 147.

[9] C. J. Horowitz and J. Piekarewicz, Phys. Rev. Lett. 86 (2001) 5647.

[10] S. Typel and B. A. Brown, Phys. Rev. C64 (2001) 027302 .

[11] F. Hofmann, C. M. Keil, and H. Lenske, Phys. Rev. C64 (2001) 034314.

[12] J. R. Stone, J. C. Miller, R. Koncewicz, P. D. Stevenson, and M. R. Strayer, Phys. Rev. C68 (2003) 034324 .

[13] A. W. Steiner and B. A. Li, Phys. Rev. C72 (2005) 041601 (R).

[14] B. A. Brown, Phys. Rev. Lett. 85 (2000) 5296.

[15] J. B. Kogut and D. K. Sinclair, Phys. Rev. D64 (2001) 034508; ibid. D66 (2002) 034505. ibid. D70 (2004) 094501.

[16] S. Gupta, hep-lat/02005; P. de Forcrand, M. A. Stephanov, and U. Wenger, PoSLAT 2007,237 ( 2007).

[17] D. T. Son and M. A. Stephanov, Phys. Rev. Lett., 86 (2001) 592.

[18] K. Splittorff, D. T. Son, and M. A. Stephanov, Phys. Rev. D64 (2001) 016003.

[19] K. Splittorff, D. Toublan, and J. J. Verbaarshot, Nucl. Phys. B639 (2002) 524.

[20] A. Barducci, R. Casalbuoni, G. Pettini, and L. Ravagli, Phys. Rev. D69 (2004) 096004; D. Ebert and K. G. Klimenko, J. Phys. G Part. Nucl. Phys. 32 (2006) 599; D. Ebert and K. G. Klimenko, Eur. Phys. J. C46 (2006) 771; S. Lawley, W. Bentz, and A. W. Thomas, Phys. Lett. B632 (2006) 495; L. He, Phys. Rev. D82 (2010) 096003.

[21] L. He, M. Jin, and P. Zhuang, Phys. Rev. D71 (2005) 116001.

[22] J. O. Andersen and L. Kyllingstad, J. Phys. G. Part. Nucl. Phys. 37 (2010) 015003.

[23] T. Sasaki, Y. Sakai, H. Kouno, and M. Yahiro, Phys. Rev. D82 (2010) 116004.

[24] J. O. Andersen, Phys. Rev. D 75, 065011 (2007); J. O. Andersen and T. Brauner, Phys. Rev. D78 (2008) 014030

[25] T. Sakai and S. Sugimoto, Prog. Theor. Phys. 113 (2005) 843; ibid. 114 (2006) 1083.

[26] D. K. Campell, R. F. Dashen, and J. T. Manassah, Phys. Rev. D12, 979 (1975); ibid. D12 (1975) 1010.

[27] S. Wolfram, The Mathematica Book, 5th edition, Wolfram Media/Cambridge University Press, Cambridge, UK, 2003.

[28] H. Abuki, R. Anglani, R. Gatto, M. Pellicoro, and M. Ruggieri, arxiv: 0809.2658.

[29] J. W. Harris and B. Muller, Ann. Rev. Nucl. Part. Sci. 46 (1996) 71.

[30] V. Bernard, Ulf- G. Meissner, and I. Zahed, Phys. Rev. D36 (1987) 819.

[31] M. Buballa, Phys. Rept. 407 (2005) 205.

[32] M. Askawa and K. Yazaki, Nucl. Phys. A504 (1989) 668.

[33] T. Hatsuda and T. Kunihiro, Phys. Rept. 247 (1994) 221.

[34] M. A. Stephanov, Int. J. Mod. Phys. A20 (2005) 4387 ; hep-ph/0402115.

[35] R. V. Gavai and S. Gupta, Phys. Rev. D68 (2003) 034506.

Received 14 October 2011. 